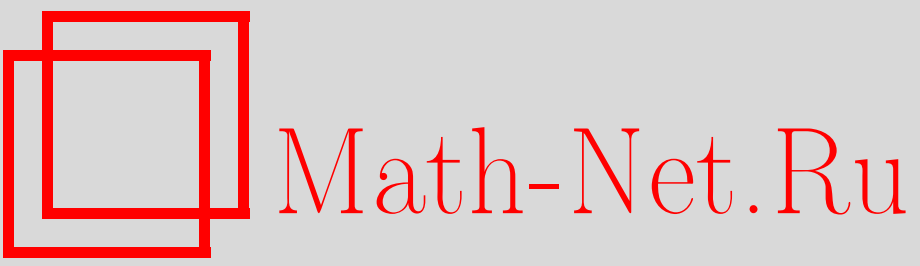

О. И. Завьялов, Еще раз о разложениях операторного произведения на световом конусе, ТМФ, 2004, том 138, номер 3, 437-452

DOI: https://doi.org/10.4213/tmf38

Использование Общероссийского математического портала Math-Net.Ru подразумевает, что вы прочитали и согласны с пользовательским соглашением

http://www . mathnet.ru/rus/agreement

Параметры загрузки:

IP : 3.85 .7 .115

26 апреля 2023 г., 13:10:40 
ТЕОРЕТИЧЕСКАЯ

И МАТЕМАТИЧЕСКАЯ

ФИЗИКА

Том 138, № 3

март, 2004

(C) 2004 г.

О.И. Завьялов

\section{ЕШЕ РАЗ О РАЗЛОЖЕНИЯХ ОПЕРАТОРНОГО ПРОИЗВЕДЕНИЯ НА СВЕТОВОМ КОНУСЕ}

Вновь рассматривается задача о разложениях на световом конусе для произведения токов. Излагаются мотивы, делающие актуальным переосмысление старых результатов.

Ключевые слова: вильсоновские разложения, разложения на световом конусе, лучевые поля, абелевы калибровочные поля.

\section{1. ВВЕДЕНИЕ}

В этой статье мы возврашаемся к задаче о разложениях на малых расстояниях и разложениях на световом конусе для произведения токов. Эта задача была решена для скалярной теории в статье [1] (см. также [2], [3]). Основным результатом этих работ стал строгий вывод соответствуюших соотношений для скалярных полей в теории возмушений с нетривиальной ультрафиолетовой перенормировкой. Процедура вывода позволила получить в явном виде правила для вычисления коэффициентов этих разложений или того, что может интерпретироваться как эти коэффициенты. Последняя оговорка сделана не случайно. Если разложения на малых расстояниях, как известно, имеют более или менее привычную форму, предсказанную Вильсоном, то вид разложений на световом конусе оказался довольно неожиданным. Оказалось, что вблизи светового конуса $T$-произведение двух токов является, так сказать, "нелокальным": оно может быть приближено линейной комбинацией интегралов по параметрам $\kappa_{1}, \ldots \kappa_{l}$ с некоторой комплексной мерой $\Psi^{\{\lambda\}}\left(\kappa_{1}, \ldots, \kappa_{l}\right)$ от нелокальных составных полей $\hat{J}_{\{\lambda\}}\left(\kappa_{1} \tilde{x}, \ldots, \kappa_{l} \tilde{x}\right)$, называемых "полями светового конуса". По существу, каждое составное поле светового конуса $\hat{J}_{\{\lambda\}}\left(\kappa_{1} \tilde{x}, \ldots, \kappa_{l} \tilde{x}\right)$ является обычным локальным составным полем $\hat{J}_{\{\lambda\}}(x)$, единственный аргумент $x$ которого распадается в каждом порядке теории возмущений на $l$ отдельных аргументов $(l-$ число внешних линий в диаграмме, представляюшей соответствующее $T$-произведение).

При этом $i$-й аргумент $x_{i}$ относится к точке $x_{i}=\kappa_{i} \tilde{x}$, лежашей на отрезке светового луча, соединяюшего начало координат с предельной точкой $\tilde{x}$ на световом конусе (таким

* Математический институт РАН им. В. А. Стеклова, Москва, Россия. E-mail: zavialov@mi.ras.ru 
образом, $\tilde{x}^{2}=0$ по определению). Поскольку параметры интегрирования, вообще говоря, непрерывны и удовлетворяют неравенствам $0<\kappa_{i}<1$, составное поле светового конуса на самом деле является нелокальным функционалом по отношению к свободному полю $\varphi(x)$.

В пределе $\kappa_{1}=\cdots=\kappa_{l}=\kappa=1$ составное поле светового конуса становится обычным локальным составным полем

$$
\hat{J}_{\{\lambda\}}(\tilde{x}, \ldots, \tilde{x})=\hat{J}_{\{\lambda\}}(\tilde{x})
$$

В свободной теории поле светового конуса просто совпадает с обычным нормальным произведением полей

$$
\hat{J}_{\{\lambda\}}\left(\kappa_{1} \tilde{x}, \ldots, \kappa_{l} \tilde{x}\right)=: \varphi_{\left(\lambda_{1}\right)}\left(\kappa_{1} \tilde{x}\right) \ldots \varphi_{\left(\lambda_{l}\right)}\left(\kappa_{l} \tilde{x}\right):
$$

где $\varphi(\lambda)$ обозначает $\lambda$-ю производную поля $\varphi$ по координате $\tilde{x}$.

Поясним причины, побудившие нас вернуться к этой давно и всесторонне изученной теме.

1. Первая причина связана с физикой. Разложения на малых расстояниях и разложения на световом конусе успешно использовались для теоретического описания так называемого глубоко неупругого рассеяния (см., например, [4]). Недавно были обнаружены [5] и другие кинематические области, допускающие применение подобной техники в рамках асимптотически свободной квантовой хромодинамики (КХД). Поэтому мы надеемся, что изложенные здесь результаты окажутся полезными для развития аналогичных методов в КХД.

2. Хорошо известно, что кроме бозонов (глюонов) в КХД рассматриваются также фермионы (кварки). Вот почему представляется необходимым распространить аргументацию, приводящую к нелокальным разложениям на световом конусе, на случай любой теории со спинорными частицами. Нам всегда казалось несомненным, что механизм появления нелокальных разложений на световом конусе является весьма обшим и отнюдь не зависит от спиновой структуры или иных свойств входящих в теорию частищ. Мы считали, что выполненный в работе [1] анализ для скалярного поля является вполне достаточной базой для обобшения доказательства на случай всякой теории, содержащей любые частицы в лагранжиане взаимодействия.

К сожалению, приведенные в работе [1] рассуждения оказались не столь ясными, как это нам казалось. Лишш одна группа теоретиков [6] предприняла серьезную попытку использовать информацию, относяшуюся к световому конусу, для описания глубоко неупругого рассеяния. Сейчас я думаю, что стремление рассмотреть в работе [1] все связанные с перенормировкой детали оказалось непродуктивным и сделало приведенное доказательство слишком трудным для понимания. Между тем ситуация с перенормировкой является достаточно очевидной. Перенормировка просто не запрещает нелокальность разложения на световом конусе. Доказательство именно этого факта в работе [1] оказалось сложным, в результате чего, вероятно, был нарушен баланс между внутренней 
ясностью логических построений и неизбежными осложнениями, связанными с перенормировкой. Вот почему в настояшей работе мы будем следовать противоположному подходу: чтобы убедить читателя в том, что нелокальность разложений на световом конусе является естественным внутренним свойством любой теории, мы сознательно проигнорируем перенормировку, принимая во внимание только нижние порядки теории возмушений, в которых нетривиальная перенормировка отсутствует.

3. Такой подход может быть применен не только в демонстрационных целях. Следует иметь в виду асимптотическую свободу КХД, что предполагает использование уравнений ренормгруппы. Поэтому позволительно иметь дело лишш с первыми порядками теории возмущений.

4. Упомянем еше один вопрос, представляюшийся важным. Совместима ли форма разложения на световом конусе, использованная в работе [1], с калибровочной инвариантностью КХД? Имеется в виду следующее. Пусть мы преобразуем левую часть разложения на световом конусе в соответствии с некоторым калибровочным преобразованием (разумеется, такая процедура вполне определена). Следует ли ожидать, что соответствуюший вид правой части сохранится при этом калибровочном преобразовании? Конечно, наиболее сингулярные члены амплитуды обнаружатся в обеих частях полученного уравнения, но возможно, что правая часть нового разложения будет включать вклады менее сингулярных членов.

Так или иначе, представляется полезным вернуться к проблеме разложений операторного произведения на световом конусе и попытаться упростить основные идеи построения в целом, уходя от непринципиальных осложнений, связанных с перенормировкой, и уделяя наибольшее внимание лишь практически важным аспектам. Эти последние иллюстрируются примерами, связанными с нижними порядками теории возмушений.

Работа будет состоять из двух частей. В данной статье мы подробно рассмотрим некоторые обшие вопросы и выполним конкретные вычисления, связанные со старшим и следуюшим за старшим членами разложений на световом конусе в рамках так называемой суперперенормируемой скалярной модели. В разделе 2 мы пересмотрим схему, приводящую к нелокальным разложениям на световом конусе для чисто скалярной теории при отсутствии ультрафиолетовых расходимостей. Чтобы проиллюстрировать основную идею, мы получим только старший член общего нелокального разложения для суперперенормируемой трилинейной скалярной модели. В отличие от работы [3], где отправной точкой служило построение так называемого оператора вычитания на световом конусе, т.е. в каком-то смысле предельного значения $T$-произведения двух токов на световом конусе, здесь мы исходим из рассмотрения $T$-произведения вне светового конуса, причем предельное значение получается автоматически. Такой подход не только выглядит более естественным. Он позволяет доказать, что (по крайней мере, в случае отсутствия ультрафиолетовых расходимостей) разложение на световом конусе может быть представлено в таком виде, что только составные поля светового конуса нулевого порядка определяют сумму соответствуюшего разложения.

Как уже отмечалось, первая модель является суперперенормируемой. Она включает 
два скалярных поля $\varphi$ и $\theta$ с лагранжианом

$$
g:[\varphi]^{2} \theta:
$$

Одной из целей нового рассмотрения этой модели является обоснование нашего базового определения $T$-произведения токов, введенного в работе [1]. Теперь скалярное поле $\varphi$ будет относиться к "кваркам", а поле $\theta$ - к упрощенным глюонам. "Кварковые" токи будут билинейными по асимптотическим полям $\varphi$ :

$$
\hat{J}(x)=S^{+} \times T\left\{:[\varphi]^{2}:(x) S\right\} .
$$

Благодаря своей суперперенормируемости эта модель имеет гораздо меньше ультрафиолетовых расходимостей и потому является гораздо более легкой для понимания, что от нее на этом этапе прежде всего и требуется. В рамках этой модели мы исследуем диаграммы двух первых порядков по константе связи. Эти диаграммы имеют ровно две внешних "кварковых" линии, поскольку именно такие диаграммы вносят вклад в асимптотическую амплитуду в обобшенном пределе Бьеркена. Среди таких диаграмм нет расходящихся, поэтому перенормировка ни в коем случае не приведет к осложнениям при работе со световым конусом.

Другая модель, называемая спинорной, будет обсуждаться во второй статье, которую нам еше предстоит написать. Будет рассмотрена абелева калибровочная теория (частной реализацией которой является квантовая электродинамика), соответствующая лагранжиану

$$
g: \bar{\psi} \gamma \psi \theta_{\mu}:
$$

где векторное поле $\theta_{\mu}$ является вкладом абелева "глюона", $\psi$ - спинорное поле (вклад кварков), $\gamma$ - некоторая матрица. Вероятно, $\gamma=\gamma_{\mu}$, в этом случае $\theta_{\mu}$ должно рассматриваться как обычный вектор; другая возможность состоит в том, что $\gamma=\gamma_{5} \gamma_{\mu}$, тогда $\theta_{\mu}$ - псевдовектор. Полная квантовая теория в этом случае содержит аномалии, что, однако, не имеет большого значения в контексте нашей задачи. Можно также думать, что $\gamma$ включает индексы цвета или запаха. Интересующие нас токи суть

$$
\begin{aligned}
& \hat{I}\left(x_{1}\right)=S^{+} \times T\left\{: \bar{\psi}\left(x_{1}\right) \gamma \psi\left(x_{1}\right): S\right\} \\
& \hat{I}\left(x_{2}\right)=S^{+} \times T\left\{: \bar{\psi}\left(x_{2}\right) \gamma \psi\left(x_{2}\right): S\right\}
\end{aligned}
$$

\section{2. ГРУППОВАЯ СТРУКТУРА $S$-МАТРИЦЫ. ПОВЕРХНОСТНЫЕ РАСХОДИМОСТИ. Т-ПРОИЗВЕДЕНИЕ ТОКОВ. РАЗЛОЖЕНИЕ НА СВЕТОВОМ КОНУСЕ ДЛЯ СКАЛЯРНОЙ МОДЕЛИ}

Этот раздел посвящен главным образом вопросу о том, почему мы в работе [1] использовали определение

$$
T\left\{\hat{J}\left(x_{1}\right) \hat{J}\left(x_{2}\right)\right\}=S^{+} \otimes T\left\{j\left(x_{1}\right) j\left(x_{2}\right) S\right\}
$$


для $T$-произведения двух токов. Наиболее простое, хотя и несколько наивное объяснение таково. Допустим, что соблюдены условия, позволяющие корректно определить так называемую "конечно-временну́ю" $S$-матрицу $S_{t_{f}, t_{i}}$ такую, что $S=S_{\infty, t} \times S_{t,-\infty}$ и $S_{t_{2}, t_{1}}^{+}=S_{t_{1}, t_{2}}$. Заметим попутно, что тогда для любого $t$

$$
S_{t_{f}, t_{i}}=S_{t_{f}, t} \otimes S_{t, t_{i}}, \quad S^{+}=S_{0,-\infty}^{+} \otimes S_{\infty, 0}^{+} .
$$

Продолжая эти “наивные" рассуждения, получим для начального $T$-произведения при $t_{1}>t_{2}$ выражение

$$
S^{+} \times S_{\infty, t_{1}} \otimes j\left(t_{1}, \mathbf{x}_{1}\right) \otimes S_{t_{1}, t_{2}} \otimes j\left(t_{2}, \mathbf{x}_{2}\right) \otimes S_{t_{2},-\infty} .
$$

Как следует из наших рассуждений, это просто $T$-произведение $T\left\{\hat{J}\left(x_{1}\right) \hat{J}\left(x_{2}\right\}\right.$. К сожалению, предположение о сушествовании "конечно-временно́й" $S$-матришы ложно для обеих упомянутых выше моделей: известно, что при наличии нетривиальной бесконечной перенормировки такая $S$-матрица содержит дополнительные специфические (так называемые "поверхностные") расходимости. С другой стороны, эта проблема может не приниматься во внимание в контексте интересующих нас нижних порядков теории возмушений, поскольку "поверхностные" расходимости сушествуют только в высших порядках. Если высшие порядки рассматриваются, естественность принятого определения $T$-произведения токов может быть обоснована с помошью условия причинности Боголюбова в дифференциальной форме. Теперь мы вернемся к обозначениям, принятым в работах [1]-[3]: с этого момента символ $T$-упорядочения операторов исчезнет из всех формул, и все произведения должны по умолчанию пониматься как $T$-упорядоченные, если только они не помечены специальным знаком, например знаком $\otimes$, уже использовавшимся вьшше (это обозначение использовалось в работах [2] и [3]).

Вернемся к обшей схеме вывода разложения на световом конусе для скалярной модели. Рассмотрим диаграмму ? в импульсном пространстве, которая вносит вклад в порядке $g^{n}$ теории возмущений для $T$-произведения двух токов $J(\xi) J(0)$. Используя $\alpha$-представление для этой диаграммы ? , получим выражение

$$
\begin{aligned}
\widetilde{?}_{2, \underline{\alpha}}\left(z_{1}, 0 \mid q_{1}, q_{2}\right)= & \text { Const } \int d \alpha_{1} \ldots d \alpha_{L} \frac{1}{A^{2}(\underline{\alpha})} \exp \{-i \mu(\underline{\alpha})\} \exp \left\{i \frac{D(\underline{\alpha})\left(z_{1}-z_{2}\right)^{2}}{4 A(\underline{\alpha})}\right\} \times \\
& \times \exp \left\{i\left(z_{1}-z_{2}\right) \sum_{j} \frac{B_{j} q_{j}}{A}\right\} \exp \{i E(\underline{q}, \underline{\alpha})\}
\end{aligned}
$$

где $z_{1}=\left\{z_{1}^{0}, \overline{z_{1}}\right\}, z_{2}=0$, а $\underline{\alpha}-L$-мерная переменная $\underline{\alpha}=\left\{\alpha_{1}, \ldots, \alpha_{L}\right\}$. Нижний индекс 2 у $\widetilde{?}$ означает, что данная диаграмма имеет ровно две внешних линии, поскольку, как уже отмечалось, только диаграммы с двумя линиями принимаются во внимание в настояшей $\mathrm{KXД.} \mathrm{Правила,} \mathrm{связываюшие} \mathrm{стандартные} \mathrm{формы} A, D, C, B_{j}$ с топологическими элементами диаграммы обшего вида, те же, что в формулах (146) из книги [3]. Сейчас эти соотношения нам не понадобятся. Функция $\mu$ есть

$$
\mu(\underline{\alpha})=\sum_{l=1}^{L} m_{l}^{2} \alpha_{l},
$$


а функция $E$ в терминах $A, B, C, D-$

$$
E=\left\{-\frac{\left(\sum_{j} B_{j} q_{j}\right)^{2}}{A D}+\frac{C}{D}+z_{2} \sum q_{j}\right\}
$$

Мы явно выпишем формы $A, B_{j}, C, D$ для конкретных примеров, которые будут рассмотрены ниже. Сейчас важно отметить, что отношение $A / D$ однородно по $\underline{\alpha}$ и индекс однородности равен 1 , а однородные функции $A$ и $B_{j}$ имеют одинаковые индексы однородности. Чтобы исследовать поведение ? около светового конуса, мы переходим к новым пространственно-временнь́м координатам каждой точки вблизи светового конуса. Именно, мы продолжаем использовать вектор $\boldsymbol{\xi}$ в качестве пространственной переменной, но используем параметр гиперболоида $\xi^{2}$ вместо временно́й координаты $\xi^{0}$. Таким образом, если, например, временна́я координата интересующей нас точки положительна, то

$$
\left\{\xi^{0}, \boldsymbol{\xi}\right\} \rightarrow\left\{+\sqrt{\xi^{2}+\boldsymbol{\xi}^{2}}, \boldsymbol{\xi}\right\}
$$

Если $\xi^{2} \rightarrow 0$, причем вектор $\boldsymbol{\xi}$ фиксирован, то предельная точка на световом конусе $\tilde{\xi}$ есть $\tilde{\xi}=\{|\boldsymbol{\xi}|, \boldsymbol{\xi}\}$.

Чтобы найти коэффициентную функцию разложения на световом конусе, перепишем выражение (5) в новых переменных:

$$
\begin{aligned}
\widetilde{?}_{2, \underline{\alpha}}\left(z_{1}^{0}, \overline{z_{1}} \mid q_{1}, q_{2}\right)= & \text { Const } \lim _{N \rightarrow \infty}\left(\int d \underline{\alpha} \frac{1}{A^{2}(\underline{\alpha})} \exp \left\{-i \frac{D(\underline{\alpha}) \xi^{2}}{4 A(\underline{\alpha})}\right\} \exp \{-i \mu(\underline{\alpha})\} \times\right. \\
& \left.\times \exp \left\{i \xi \frac{B_{1} q_{1}+B_{2} q_{2}}{A}\right\}\left\{1+\sum_{n=1}^{N} \frac{i^{n} E^{n}}{n !}\right\}\right) .
\end{aligned}
$$

Теперь разложим подынтегральное выражение в (7) в степенной ряд по $\xi^{2}$, используя соотношение

$$
\sqrt{\boldsymbol{\xi}^{2}+\xi^{2}}=|\boldsymbol{\xi}|\left\{1+\frac{\xi^{2}}{2|\boldsymbol{\xi}|^{2}} \sum_{n=2}^{\infty} \frac{(-1)^{n+1}(2 n-3) ! !}{(2)^{n} n !}\left(\frac{\xi}{|\boldsymbol{\xi}|}\right)^{2 n}\right\}
$$

Таким образом,

$$
\begin{aligned}
\widetilde{?}_{2, \underline{\alpha}}\left(z_{1}^{0}, \overline{z_{1}} \mid q_{1}, q_{2}\right)=\text { Const } \lim _{N \rightarrow \infty}\left(\int d \underline{\alpha} \frac{1}{A^{2}(\underline{\alpha})} \exp \left\{-i \frac{D(\underline{\alpha}) \xi^{2}}{4 A(\underline{\alpha})}\right\} \exp \{-i \mu(\underline{\alpha})\} \times\right. \\
\quad \times \exp \left\{i\left(|\boldsymbol{\xi}|+\frac{\xi^{2}}{2|\boldsymbol{\xi}|}+\sum_{n=2}^{N} \frac{(-1)^{n+1}(2 n-3) ! !}{(2)^{n} n !} \frac{\xi^{2 n}}{|\boldsymbol{\xi}|^{2 n-1}}\right) \frac{B_{1} q_{1}^{(0)}+B_{2} q_{2}^{(0)}}{A}\right\} \times \\
\left.\quad \times \exp \left\{-i \boldsymbol{\xi} \frac{B_{1} \mathbf{q}_{1}+B_{2} \mathbf{q}_{2}}{A}\right\}\left\{1+\sum_{n=1}^{N} \frac{i^{n} E^{n}}{n !}\right\}\right) .
\end{aligned}
$$


Чтобы вычислить старший член разложения на световом конусе, соответствуюшего данной диаграмме $\widetilde{?}$, достаточно удержать лишь первые слагаемые из бесконечного степенного ряда, заключенного в фигурные скобки в уравнении (8). Имеем

$$
\begin{aligned}
\widetilde{?}_{2, \underline{\alpha}}^{(\mathrm{L})}\left(z_{1}^{0}, \overline{z_{1}} \mid q_{1}, q_{2}\right)= & \text { Const } \int d \underline{\alpha} \frac{1}{A^{2}(\underline{\alpha})} \exp \left\{-i \frac{D(\underline{\alpha}) \xi^{2}}{4 A(\underline{\alpha})}\right\} \exp \{-i \mu(\underline{\alpha})\} \times \\
& \times \exp \left\{i \tilde{\xi} \frac{B_{1} q_{1}+B_{2} q_{2}}{A}\right\} .
\end{aligned}
$$

Новое обозначение (L) в ? ${ }^{(\mathrm{L})}$ сигнализирует о том, что принимается во внимание только старшая сингулярность по отношению к $\xi^{2}: \xi^{2}$ удерживается только в первой экспоненте, а во всех остальных местах $\xi^{2}$ полагается равным 0. Если говорить о бесконечной сумме внутри первой пары фигурных собок в уравнении (8), то это вполне понятно: фактор $\xi^{2}$ содержится лишь в тех членах этой бесконечной суммы, которые пренебрегаются рассматриваемым здесь приближением. Именно эти члены, ответственные за "сглаживание" сингулярности, делают ее более "регулярной". Иными словами, старшее приближение не зависит от опушенной части ряда. Ниже мы покажем, что аналогичная замена бесконечной суммы конечной внутри второй пары фигурных скобок также не влияет на старшую сингулярность, хотя приводящий к этому конкретный механизм несколько иной.

Теперь нужно вернуться к координатной коэффициентной функции

$$
?_{2, \underline{\alpha}}^{(\mathrm{L})}\left(\xi^{2}, \boldsymbol{\xi} \mid y_{1}, y_{2}\right)=\frac{1}{(2 \pi)^{8}} \text { Const } \int d q_{1} d q_{2} \exp \left\{-i y_{1} q_{1}-i y_{2} q_{2}\right\} \widetilde{?}_{2, \underline{\alpha}}^{(\mathrm{L})}\left(\xi^{2}, \boldsymbol{\xi} \mid q_{1}, q_{2}\right) \text {. }
$$

Нетрудно убедиться, что сказанное эквивалентно следуюшему утверждению: старшее приближение для разложения на световом конусе, соответствуюшего диаграмме $g^{n}$, может быть представлено в виде

$$
G^{(\mathrm{L})}(\xi)=g^{n} \int d \kappa_{1} d \kappa_{2} \Psi\left(\kappa_{1}, \kappa_{2}\right) J\left(\kappa_{1}, \kappa_{2} \mid \tilde{\xi}\right)+Q(\xi),
$$

где остаточный член $Q(\xi)$ менее сингулярен, чем $G(\xi)$ при $\xi \rightarrow \tilde{\xi}$ на световом конусе. Составное поле светового конуса $J\left(\kappa_{1}, \kappa_{2} \mid \tilde{\xi}\right)$ является билинейным по свободному полю $\varphi(x)$ и есть просто нормальное произведение двух таких полей:

$$
J\left(\kappa_{1}, \kappa_{2} \mid \tilde{\xi}\right)=: \varphi\left(\kappa_{1} \tilde{\xi}\right) \varphi\left(\kappa_{2} \tilde{\xi}\right): .
$$

Комплексная мера $\Psi\left(\kappa_{1}, \kappa_{2}\right)$, генерируемая диаграммой $g^{n} ?$, равна интегралу

$$
\Psi\left(\xi^{2} \mid \kappa_{1}, \kappa_{2}\right)=\text { Const } \int d \underline{\alpha} \frac{1}{A^{2}} \exp \left\{-i \frac{D \xi^{2}}{4 A}\right\} \delta\left(\kappa_{1}-\frac{B_{1}}{A}\right) \delta\left(\kappa_{2}-\frac{B_{2}}{A}\right) .
$$

Для завершения доказательства мы должны показать, что приближение, связанное с заменой

$$
\exp \left\{-i \frac{\left(\sum_{j} B_{j} q_{j}\right)^{2}}{A D}+i \frac{C}{D}-i \mu(\underline{\alpha})+i z_{2} \sum q_{j}\right\} \rightarrow 1
$$


в (5), не влияет на старший член разложения на световом конусе. Прежде всего, $z_{2}=0$ по нашему исходному предположению, поэтому соответствующий множитель ни на что не влияет. Далее, вспомним о так называемых переменных Хеппа $t_{l}$, которые задают область интегрирования в исходном $\alpha$-представлении в виде объединения секторов Хеппа вида $\alpha_{1}<\alpha_{2}<\cdots<\alpha_{L}$ при всех возможных порядках нумерации. Переход к этим переменным заметно упрошает структуру сингулярностей во всех типичных подынтегральных выражениях.

Только одна из переменных $t_{l}$, построенных таким образом, а именно $t=t_{L}$, меняется в бесконечных пределах $0, \infty$ (номер $L$ может быть представлен произвольной линией диаграммы), а все остальные $t_{l}(l<L)$ остаются конечными: $0<t_{l}<1$. Посмотрим теперь, каким образом единственная бесконечная переменная $t$ входит во вклад от каждого сектора Хеппа в интеграл (5). Обозначим этот вклад $G_{s}$. Имеем

$$
G_{s}=\int_{0}^{\infty} \frac{d t}{t^{(2-w)} a^{2}} \int_{0}^{1} d \nu\left(\underline{t}_{l}\right) \exp \left\{-i \frac{\xi^{2}}{4 t a}\right\} E(t F) \Phi
$$

где функции $d, a, F$ и $\Phi$ не зависят от $t$ и $E(0)=1$, а $w$ есть некоторое целое число $(\nu$ - мера для параметров, меняюшихся в конечных пределах). Случай, когда $E$ меняется на 1, уже был рассмотрен. Рассмотрим теперь ситуацию, когда какая-то другая часть $E$, например $t^{n} E_{n}$, где $E_{n}$ не зависит от $t$, оказывается существенной в подынтегральном выражении в (14). Нетрудно видеть, что появление дополнительного множителя $t$ под знаком интеграла в (13) эквивалентно умножению функционала на световом конусе на дополнительную “сглаживаюшую” функцию $\xi^{2 n}$. Достаточно выполнить замену переменных

$$
t \rightarrow \tau=\frac{t}{\xi^{2}}
$$

в (13). Появление такой “сглаживающей” функции перед интегралом означает, что последний не может представлять старший член.

\section{3. СКАЛЯРНАЯ МОДЕЛЬ}

В свободном приближении скалярной модели, т.е. в нулевом порядке по $g$, имеется нетривиальная диаграмма с двумя внешними $\varphi$-линиями, поскольку в силу теоремы Вика необходимо учесть причинную функцию Грина $D^{\mathrm{c}}$, связывающую координаты $x_{1}=\xi$ и $x_{2}=0$. Другими словами, $T$-произведение двух токов есть

$$
(J(\xi) J(0))_{+}=F(\xi)=4 D^{\mathrm{c}}(\xi): \varphi(0) \varphi(\xi):
$$

Мы переходим к построению остаточного члена в разложении на световом конусе, соответствующем единственной существенной диаграмме порядка $g^{0}$. Введем обозначение

$$
Q(\xi)=4 D^{\mathrm{c}}(\xi): \varphi(0)\left[\varphi(\xi)-\varphi(\tilde{\xi})-\left.\left\{\frac{d}{d l^{0}} \varphi(l)\right\}\right|_{l=\tilde{\xi}}\left(\frac{1}{2} \frac{\xi^{2}}{\sqrt{\xi^{2}}}\right)\right]:
$$


В используемых нами сейчас переменных $\{\xi\}=\left\{\sqrt{\xi^{2}+\xi^{2}}, \xi\right\}$ при $\xi^{2} \rightarrow 0$ (т.е. при $\xi \rightarrow$ $\tilde{\xi})$ выражение в квадратных скобках пропорционально $\left(\xi^{2}\right)^{2}$, а самый сингулярный член в $D^{\mathrm{c}}(\xi)$ имеет полюс $\xi^{(-2)}$. Следовательно, остаточный член $Q(\xi)$ стремится к нулю при $\xi \rightarrow \tilde{\xi}$. Помимо этого члена остается выражение

$$
(J(\xi) J(0))_{+} \backsim 4 D^{\mathrm{c}}(\xi): \varphi(0)\left[\varphi(\tilde{\xi})+\left.\left\{\frac{d}{d l^{0}} \varphi(l)\right\}\right|_{l=\tilde{\xi}}\left(\frac{1}{2} \frac{\xi^{2}}{\sqrt{\xi^{2}}}\right)\right]: .
$$

Действительно, имеет место тождество

$$
\left(J(\xi J(0))_{+}=Q(\xi)+4 D^{\mathrm{c}}(\xi): \varphi(0)\left[\varphi(\tilde{\xi})+\left.\left\{\frac{d}{d l^{0}} \varphi(l)\right\}\right|_{l=\tilde{\xi}}\left(\frac{1}{2} \frac{\xi^{2}}{\sqrt{\xi^{2}}}\right)\right]: .\right.
$$

Поскольку, как мы уже знаем, первый член сушественно не влияет на поведение этого выражения по отношению к $\xi^{2}$, мы будем интерпретировать его как малый остаточный вклад. Второй же член приводит к предельному функционалу в виде интеграла полей светового конуса по параметрам $\kappa_{1}, \kappa_{2}$ :

$$
F^{\lim }(\xi)=\int_{0}^{1} d \kappa_{1} d \kappa_{2}\left[\Psi_{(0)}^{\prime}\left(\xi^{2} \mid \kappa_{1}, \kappa_{2}\right) J_{(0)}^{\prime}\left(\kappa_{1}, \kappa_{2}, \boldsymbol{\xi}\right)+\Psi_{(0)}^{\prime \prime}\left(\xi^{2} \mid \kappa_{1}, \kappa_{2}\right) J_{(0)}^{\prime \prime}\left(\kappa_{1}, \kappa_{2}, \boldsymbol{\xi}\right)\right] .
$$

Два слагаемых в этом выражении соответствуют разным составным полям светового конуса $J^{\prime}$ и $J^{\prime \prime}$. Первое из них (или, лучше сказать, мера $\Psi^{\prime}$ ) ответственно за главную сингулярность:

$$
\begin{aligned}
\Psi_{(0)}^{\prime}\left(\xi^{2} \mid \kappa_{1}, \kappa_{2}\right) & =\delta\left(\kappa_{1}\right) \delta\left(\kappa_{2}-1\right) 4 D^{\mathrm{c}}(\xi), \\
J_{(0)}^{\prime}\left(\kappa_{1}, \kappa_{2}, \boldsymbol{\xi}\right) & =: \varphi\left(\kappa_{1} \tilde{\xi}\right) \varphi\left(\kappa_{2} \tilde{\xi}\right):
\end{aligned}
$$

а второе слагаемое - за сингулярность, следуюшую за главной:

$$
\begin{aligned}
\Psi_{(0)}^{\prime \prime}\left(\xi^{2} \mid \kappa_{1}, \kappa_{2}\right) & =2 D^{\mathrm{c}}(\xi) \xi^{2} \delta\left(\kappa_{1}\right) \delta\left(\kappa_{2}-1\right), \\
J_{(0)}^{\prime \prime}\left(\kappa_{1}, \kappa_{2}, \boldsymbol{\xi}\right) & =\frac{1}{|\boldsymbol{\xi}|}: \varphi\left(\kappa_{1} \tilde{\xi}\right)\left[\frac{d}{d l^{0}} \varphi(l)\right]:\left.\right|_{l=\kappa_{2} \tilde{\xi}} .
\end{aligned}
$$

Чтобы перейти к более общему и распространенному языку $\alpha$-представления, мы переформулируем эти результаты следуюшим образом. Координатная коэффишиентная функция функционала (16) есть

$$
\widetilde{?}_{2}\left(\xi^{2}, \boldsymbol{\xi} \mid y_{1}, y_{2}\right)=4 D^{\mathrm{c}}(\xi) \delta\left(y_{1}-\xi\right) \delta\left(y_{2}\right) .
$$

Легко проверить, что коэффициентная функция (25) в точности соответствует функционалу (16).Коэффициентная функция этого функционала в импульсном пространстве есть

$$
\widetilde{?}_{2}\left(\xi^{2}, \boldsymbol{\xi} \mid q_{1}, q_{2}\right)=\int d y_{1} d y_{2} \exp \left\{i q_{1} y_{1}+i q_{2} y_{2}\right\} ?_{2}\left(\xi^{2}, \boldsymbol{\xi} \mid y_{1}, y_{2}\right)=4 D^{\mathrm{c}}(\xi) \exp \left\{i q_{1} \xi\right\}
$$


Чтобы перейти к $\alpha$-представлению, вспомним, что для рассматриваемой нами конкретной диаграммы

$$
D(\underline{\alpha})=1, \quad A=\alpha, \quad B_{1}=\alpha, \quad B_{2}=0, \quad C=\alpha q_{1}^{2},
$$

так что $E=0$. Далее мы продолжаем рассмотрение в $\alpha$-представлении:

$$
\widetilde{?}_{2, \underline{\alpha}}\left(\xi^{2}, \boldsymbol{\xi} \mid q_{1}, q_{2}\right)=\text { Const } \int_{0}^{\infty} \frac{d \alpha}{\alpha^{2}} \exp \left\{i \frac{\xi^{2}}{4 \alpha}\right\} \exp \{-i \mu(\underline{\alpha})\} \exp \left\{i \xi q_{1}\right\}
$$

На этот раз $\mu(\underline{\alpha})=m^{2} \alpha$. Чтобы воспроизвести два члена в предельном значении функционала $F(\xi)$, старший и следуюший за старшим, достаточно удержать лишь главные вклады в бесконечный степенной ряд, представляющий вектор $\xi$. Другими словами,

$$
\begin{aligned}
\widetilde{\tau}{ }_{2, \underline{\alpha}}^{\lim }\left(\xi^{2}, \boldsymbol{\xi} \mid q_{1}, q_{2}\right)= & \text { Const } \int_{0}^{\infty} \frac{d \alpha}{\alpha^{2}} \exp \left\{i \frac{\xi^{2}}{4 \alpha}\right\} \exp \{-i \mu(\underline{\alpha})\} \times \\
& \times \exp \left\{i q_{1}^{0}\left[|\boldsymbol{\xi}|+\frac{\xi^{2}}{2|\boldsymbol{\xi}|}+\cdots\right]\right\} \exp \left\{-i \boldsymbol{\xi} \mathbf{q}_{1}\right\}
\end{aligned}
$$

Для координатного представления найдем

$$
\begin{aligned}
?_{2, \underline{\alpha}}^{\lim }\left(\xi^{2}, \boldsymbol{\xi} \mid y_{1}, y_{2}\right)= & \text { Const } \int_{0}^{\infty} \frac{d \alpha}{\alpha^{2}} \exp \left\{i \frac{\xi^{2}}{4 \alpha}\right\} \exp \{-i \mu(\underline{\alpha})\} \times \\
& \times \delta\left(y_{1}^{0}-|\boldsymbol{\xi}|-\frac{\xi^{2}}{2|\boldsymbol{\xi}|}\right) \delta\left(\mathbf{y}_{\mathbf{1}}-\boldsymbol{\xi}\right) \delta\left(y_{2}\right),
\end{aligned}
$$

что возвращает нас к уравнению (20).

Обратимся теперь к высшим порядкам теории возмушений. Рассмотрим диаграмму, которая состоит из четырех внешних линий. Этой диаграмме также соответствует нетривиальный вклад в функционал $G(\xi)=(J(\xi) J(0))_{+}$. В этом случае $\alpha$-формы суть

$$
\begin{gathered}
D=\alpha_{0}+\alpha_{1}+\alpha_{2}+\alpha_{3} ; \quad A=\alpha_{0}\left(\alpha_{1}+\alpha_{2}+\alpha_{3}\right) ; \\
B_{1}=\alpha_{0}\left(\alpha_{2}+\alpha_{3}\right) ; \quad B_{2}=\alpha_{0} \alpha_{2} ; \\
C=\left(q_{1}+q_{2}\right)^{2} \alpha_{0} \alpha_{2}+q_{1}^{2} \alpha_{0} \alpha_{3} ; \quad \mu(\underline{\alpha})=m^{2}\left(\alpha_{0}+\alpha_{1}+\alpha_{2}\right)+M^{2} \alpha_{3} .
\end{gathered}
$$

Коэффициентная функция $\widetilde{G}_{2, \underline{\alpha}}\left(\xi^{2}, \boldsymbol{\xi} \mid q_{1}, q_{2}\right)$ соответствующего функционала $G(\xi)$ есть

$$
\begin{aligned}
\widetilde{G}_{2, \underline{\alpha}}\left(\xi^{2}, \boldsymbol{\xi} \mid q_{1}, q_{2}\right)= & \text { Const } \int \frac{d \underline{\alpha}}{\alpha_{0}^{2}\left(\alpha_{1}+\alpha_{2}+\alpha_{3}\right)^{2}} \exp \left\{i \frac{\left(\alpha_{0}+\alpha_{1}+\alpha_{2}+\alpha_{3}\right) \xi^{2}}{4 \alpha_{0}\left(\alpha_{1}+\alpha_{2}+\alpha_{3}\right)}\right\} \times \\
& \times \exp \{-i \mu(\underline{\alpha})\} \exp \left\{i \xi^{0}\left(\xi^{2}, \boldsymbol{\xi}\right) \frac{\left(\alpha_{2}+\alpha_{3}\right) q_{1}^{0}+\alpha_{2} q_{2}^{0}}{\alpha_{1}+\alpha_{2}+\alpha_{3}}\right\} \times \\
& \times \exp \left\{-i \boldsymbol{\xi} \frac{\left(\alpha_{2}+\alpha_{3}\right) \mathbf{q}_{1}+\alpha_{2} \mathbf{q}_{2}}{\alpha_{1}+\alpha_{2}+\alpha_{3}}\right\} \exp \{i E\} .
\end{aligned}
$$


Начиная исследовать первую нетривиальную “динамическую" диаграмму, стоит заметить, что сингулярность “динамического" функционала $G(\xi)$ как таковая может не иметь ничего общего с сингулярностью свободного функционала $F(\xi)$. Действительно, сингулярность “динамического” фукционала, несомненно, должна существенно зависеть от характера (супер)перенормируемости теории. Мы еще вернемся к этому при рассмотрении спинорной теории. Введем обозначения

$$
\begin{aligned}
\kappa_{1} & =\frac{\alpha_{2}+\alpha_{3}}{\alpha_{1}+\alpha_{2}+\alpha_{3}}, \\
\kappa_{2} & =\frac{\alpha_{2}}{\alpha_{1}+\alpha_{2}+\alpha_{3}} .
\end{aligned}
$$

Почти так же, как это было сделано вьше, мы вычислим два главных вклада, старший и следующий за старшим, от функционала (32) в предельное значение $G^{\lim }(\xi)$. В соответствии с предположениями, сделанными в начале этой статьи, высшие приближения к разложению на световом конусе, содержашие "сглаживаюшие" функции, возникают из двух источников: $G^{\lim }=G^{\prime}+G^{\prime \prime}$. Вклад $G^{\prime}$ появляется, если мы удерживаем второй член бесконечного степенного ряда, представляюшего вектор $\xi_{0}=\sqrt{|\boldsymbol{\xi}|^{2}+\xi^{2}}=|\boldsymbol{\xi}|+$ $\xi^{2} /(2|\boldsymbol{\xi}|)+\cdots$. Повторяя рассуждения, приведшие нас к формуле (30), легко восстановить член $G^{\prime}$, т.е. интересующий нас вклад:

$$
\begin{aligned}
\widetilde{G}_{2, \underline{\alpha}}^{\prime}\left(\xi^{2}, \boldsymbol{\xi} \mid q_{1}, q_{2}\right)= & \text { Const } \int \frac{d \underline{\alpha}}{A^{2}(\underline{\alpha})} \exp \left\{i \frac{D \xi^{2}}{4 A}\right\} \exp \{-i \mu(\underline{\alpha})\} \times \\
& \times \exp i\left\{|\boldsymbol{\xi}|+\frac{\xi^{2}}{2|\boldsymbol{\xi}|}\right\}\left(\kappa_{1} q_{1}^{0}+\kappa_{2} q_{2}^{0}\right) \exp \left\{-i \boldsymbol{\xi}\left(\kappa_{1} \mathbf{q}_{1}+\kappa_{2} \mathbf{q}_{2}\right)\right\} .
\end{aligned}
$$

Этим, однако, вопрос не исчерпьвается. Чтобы получить всю необходимую информацию, нужно еше прибавить к $\widetilde{G}_{2, \underline{\alpha}}^{\prime}\left(\xi^{2}, \boldsymbol{\xi} \mid q_{1}, q_{2}\right)$ член $\widetilde{G}_{2, \underline{\alpha}}^{\prime \prime}\left(\xi^{2}, \boldsymbol{\xi} \mid q_{1}, q_{2}\right)$. Теперь вклад $G^{\prime \prime}$ связан просто со второй (следуюшей за главной) частью бесконечной суммы, а именно с суммой, представляющей множитель $\exp \{i E\}$ в формуле (32). Мы утверждаем, что необходимая точность будет достигнута, если в $G^{\prime \prime}$ удержать только один дополнительньй член $(i E)$ из этой суммы:

$$
\begin{aligned}
\widetilde{G}_{2, \underline{\alpha}}^{\prime \prime}\left(\xi^{2}, \boldsymbol{\xi} \mid q_{1}, q_{2}\right)= & \text { Const } \int \frac{d \underline{\alpha}}{A^{2}(\underline{\alpha})} \exp \left\{i \frac{D \xi^{2}}{4 A}\right\} \times \\
& \times \exp \{-i \mu(\underline{\alpha})\}\{i E\} \exp \left\{i \tilde{\xi}\left(\kappa_{1} q_{1}+\kappa_{2} q_{2}\right)\right\} .
\end{aligned}
$$

Другими словами, основное утверждение состоит в том, что величина $Q(\xi)=G(\xi)-$ $G^{\lim }(\xi)=G(\xi)-G^{\prime}(\xi)-G^{\prime \prime}(\xi)$ является менее сингулярной (порядка $\left.\left(\xi^{2}\right)^{2}\right)$ при $\xi \rightarrow \tilde{\xi}$, чем сама величина $G(\xi)$. Подтверждаюшая это аргументация будет приведена в конце этого раздела. Теперь мы продолжаем следовать стандартной схеме рассуждений. Можно написать

$$
G(\xi)=Q(\xi)+G^{\lim }(\xi)
$$


что лишш выражает тождественную операцию. Поскольку с необходимой точностью можно считать $Q(\xi)$ малым остаточным членом, остается только показать, что можно представить $G^{\lim }(\xi)$ в виде конечной суммы интегралов от полей светового конуса. Это можно сделать вполне рутинным образом.

Прежде всего заметим, что $E$ квадратично по $q_{1}$ и $q_{2}: E=E_{11} q_{1}^{2}+E_{22} q_{2}^{2}+E_{12} q_{1} q_{2}$. Более того, $E_{i j}$ суть однородные (порядка 1 ) функции по отношению к $\underline{\alpha}$. Например,

$$
E_{12}=\frac{2 \alpha_{0} \alpha_{1} \alpha_{2}}{\left(\alpha_{0}+\alpha_{1}+\alpha_{2}+\alpha_{3}\right)\left(\alpha_{1}+\alpha_{2}+\alpha_{3}\right)}
$$

и т.д.

Возвращаясь к координатному представлению, найдем

$$
\begin{aligned}
G_{2, \underline{\alpha}}^{\prime}\left(\xi^{2}, \boldsymbol{\xi} \mid y_{1}, y_{2}\right)= & \text { Const } \int \frac{d \underline{\alpha}}{A^{2}(\underline{\alpha})} \exp \left\{i \frac{D \xi^{2}}{4 A}\right\} \exp \{-i \mu(\underline{\alpha})\} \times \\
& \times\left(\delta\left(y_{1}^{0}-\kappa_{1}\left[|\boldsymbol{\xi}|+\frac{\xi^{2}}{2|\boldsymbol{\xi}|}\right]\right) \delta\left(\mathbf{y}_{1}-\kappa_{1} \boldsymbol{\xi}\right) \times\right. \\
& \left.\times \delta\left(y_{2}^{0}-\kappa_{2}\left[|\boldsymbol{\xi}|+\frac{\xi^{2}}{2|\boldsymbol{\xi}|}\right]\right) \delta\left(\mathbf{y}_{2}-\kappa_{2} \boldsymbol{\xi}\right)\right) \\
G_{2, \underline{\alpha}}^{\prime \prime}\left(\xi^{2}, \boldsymbol{\xi} \mid y_{1}, y_{2}\right)= & \text { Const } \int \frac{d \underline{\alpha}}{A^{2}(\underline{\alpha})} \exp \left\{i \frac{D \xi^{2}}{4 A}\right\} \exp \{-i \mu(\underline{\alpha})\} \times \\
& \times(-i)\left[E_{11}(\underline{\alpha}) \frac{d^{2}}{\left(d y_{1}\right)^{2}}+E_{22} \frac{d^{2}}{\left(d y_{2}\right)^{2}}+E_{12} \frac{d^{2}}{d y_{1} d y_{2}}\right] \times \\
& \times \delta\left(y_{1}-\kappa_{1} \tilde{\xi}\right) \delta\left(y_{2}-\kappa_{2} \tilde{\xi}\right) .
\end{aligned}
$$

Таким образом, получаем

$$
\begin{aligned}
G_{2, \underline{\alpha}}^{\lim }\left(\xi^{2}, \boldsymbol{\xi} \mid y_{1}, y_{2}\right)= & G_{2, \underline{\alpha}}^{\prime}\left(\xi^{2}, \boldsymbol{\xi} \mid y_{1}, y_{2}\right)+G_{2, \underline{\alpha}}^{\prime \prime}\left(\xi^{2}, \boldsymbol{\xi} \mid y_{1}, y_{2}\right)= \\
= & \operatorname{Const} \int \frac{d \underline{\alpha}}{A^{2}(\underline{\alpha})} \exp \left\{i \frac{D \xi^{2}}{4 A}\right\} \exp \{-i \mu(\underline{\alpha})\} \times \\
& \times\left(\delta\left(y_{1}^{0}-\kappa_{1}\left[|\boldsymbol{\xi}|+\frac{\xi^{2}}{2|\boldsymbol{\xi}|}\right]\right) \delta\left(\mathbf{y}_{1}-\kappa_{1} \boldsymbol{\xi}\right) \times\right. \\
& \left.\times \delta\left(y_{2}^{0}-\kappa_{2}\left[|\xi|+\frac{\xi^{2}}{2|\xi|}\right]\right) \delta\left(\mathbf{y}_{2}-\kappa_{2} \boldsymbol{\xi}\right)\right)+ \\
& +(-i)\left[E_{11}(\underline{\alpha}) \frac{d^{2}}{\left(d y_{1}\right)^{2}}+E_{22} \frac{d^{2}}{\left(d y_{2}\right)^{2}}+E_{12} \frac{d^{2}}{d y_{1} d y_{2}}\right] \times \\
& \times \delta\left(y_{1}-\kappa_{1} \tilde{\xi}\right) \delta\left(y_{2}-\kappa_{2} \tilde{\xi}\right) .
\end{aligned}
$$


Это значит, что “предельный” функционал допускает представление

$$
\begin{aligned}
G_{2, \underline{\alpha}}^{\lim }\left(\xi^{2}, \boldsymbol{\xi} \mid y_{1}, y_{2}\right)= & G_{2, \underline{\alpha}}^{\prime}\left(\xi^{2}, \boldsymbol{\xi} \mid y_{1}, y_{2}\right)+G_{2, \underline{\alpha}}^{\prime \prime}\left(\xi^{2}, \boldsymbol{\xi} \mid y_{1}, y_{2}\right)= \\
= & \text { Const } \int \frac{d \underline{\alpha}}{A^{2}(\underline{\alpha})} \exp \left\{i \frac{D \xi^{2}}{4 A}\right\} \exp \{-i \mu(\underline{\alpha})\} \times \\
& \times\left(\delta\left(y_{1}^{0}-\kappa_{1}\left[|\boldsymbol{\xi}|+\frac{\xi^{2}}{2|\boldsymbol{\xi}|}\right]\right) \delta\left(\mathbf{y}_{1}-\kappa_{1} \boldsymbol{\xi}\right) \times\right. \\
& \left.\times \delta\left(y_{2}^{0}-\kappa_{2}\left[|\xi|+\frac{\xi^{2}}{2|\xi|}\right]\right) \delta\left(\mathbf{y}_{2}-\kappa_{2} \boldsymbol{\xi}\right)\right)+ \\
& +(-i)\left[E_{11}(\underline{\alpha}) \frac{d^{2}}{\left(d y_{1}^{0}\right)^{2}}+E_{22} \frac{d^{2}}{\left(d y_{2}^{0}\right)^{2}}+E_{12} \frac{d^{2}}{d y_{1}^{0} d y_{2}^{0}}\right] \times \\
& \times \delta\left(y_{1}-\kappa_{1} \tilde{\xi}\right) \delta\left(y_{2}-\kappa_{2} \tilde{\xi}\right)
\end{aligned}
$$

с необходимой нам точностью. Окончательно,

$$
\begin{aligned}
G^{\lim }(\xi)= & \text { Const } \int \frac{d \underline{\alpha}}{A^{2}(\underline{\alpha})} \exp \left\{i \frac{D \xi^{2}}{4 A}\right\} \exp \{-i \mu(\underline{\alpha})\} \times \\
& \times\left(: \varphi\left(\kappa_{1} \tilde{\xi}\right) \varphi\left(\kappa_{2} \tilde{\xi}\right):+\frac{\kappa_{1} \xi^{2}}{2|\xi|}: \frac{d \varphi(l)}{d l^{0}} \varphi\left(\kappa_{2} \tilde{\xi}\right):\left.\right|_{l=\kappa_{2} \tilde{\xi}}+\right. \\
& +\frac{\kappa_{2} \xi^{2}}{2|\xi|}: \varphi\left(\kappa_{1} \tilde{\xi}\right) \frac{d \varphi(q)}{d q^{0}}:\left.\right|_{q=\kappa_{2} \tilde{\xi}}+(-i)\left[E_{11}:\left.\frac{d^{2} \varphi(l)}{(d l)^{2}}\right|_{l=\kappa_{1} \tilde{\xi}} \varphi\left(\kappa_{2} \tilde{\xi}\right):+\right. \\
& \left.\left.+E_{22}: \varphi\left(\kappa_{1} \tilde{\xi}\right) \frac{d^{2} \varphi(q)}{(d q)^{2}}:\left.\right|_{q=\kappa_{2} \tilde{\xi}}+E_{12}: \frac{d \varphi(l)}{d l} \frac{d \varphi(q)}{d q}:\left.\right|_{l=\kappa_{1} \tilde{\xi} q=\kappa_{2} \tilde{\xi}}\right]\right) .
\end{aligned}
$$

Как станет ясно позже, когда мы определим так называемую “барицентрическую” замену переменных, последнее выражение легко преобразовать в нужную нам сумму интегралов от полей светового конуса:

$$
\begin{aligned}
G^{\lim }(\xi)= & \int d \kappa_{1} d \kappa_{2}\left(\Psi^{(\mathrm{L})}\left(\xi^{2} \mid \kappa_{1}, \kappa_{2}\right) J^{(\mathrm{L})}\left(\kappa_{1}, \kappa_{2} \mid \boldsymbol{\xi}\right)+\Psi^{\prime}\left(\xi^{2} \mid \kappa_{1}, \kappa_{2}\right) J^{\prime}\left(\kappa_{1}, \kappa_{2} \mid \boldsymbol{\xi}\right)+\right. \\
& +\Psi^{\prime \prime}\left(\xi^{2} \mid \kappa_{1}, \kappa_{2}\right) J^{\prime \prime}\left(\kappa_{1}, \kappa_{2} \mid \boldsymbol{\xi}\right)+\Psi_{11}\left(\xi^{2} \mid \kappa_{1}, \kappa_{2}\right) J_{11}\left(\kappa_{1}, \kappa_{2} \mid \boldsymbol{\xi}\right)+ \\
& \left.+\Psi_{22}\left(\xi^{2} \mid \kappa_{1}, \kappa_{2}\right) J_{22}\left(\kappa_{1}, \kappa_{2} \mid \boldsymbol{\xi}\right)+\Psi_{12}\left(\xi^{2} \mid \kappa_{1}, \kappa_{2}\right) J_{12}\left(\kappa_{1}, \kappa_{2} \mid \boldsymbol{\xi}\right)\right)
\end{aligned}
$$

В этом уравнении $\Psi^{(\mathrm{L})}\left(\xi^{2} \mid \kappa_{1}, \kappa_{2}\right)$ и $J^{(\mathrm{L})}\left(\kappa_{1}, \kappa_{2} \mid \boldsymbol{\xi}\right)$ представляют главный член разложения на световом конусе:

$$
\begin{aligned}
\Psi^{(\mathrm{L})}\left(\xi^{2} \mid \kappa_{1}, \kappa_{2}\right)= & \text { Const } \int \frac{d \underline{\alpha}}{A^{2}(\underline{\alpha})} \exp \left\{i \frac{D \xi^{2}}{4 A}\right\} \exp \{-i \mu(\underline{\alpha})\} \times \\
& \times \delta\left(\kappa_{1}-\frac{\alpha_{2}+\alpha_{3}}{\alpha_{1}+\alpha_{2}+\alpha_{3}}\right) \delta\left(\kappa_{2}-\frac{\alpha_{2}}{\alpha_{1}+\alpha_{2}+\alpha_{3}}\right), \\
J^{(\mathrm{L})}\left(\kappa_{1}, \kappa_{2} \mid \xi\right)= & : \varphi\left(\kappa_{1} \tilde{\xi}\right) \varphi\left(\kappa_{2} \tilde{\xi}\right): .
\end{aligned}
$$

4 Теоретическая и математическая физика, т. 138, № 3, 2004 г. 
Другие составляющие уравнения (43) представляют следующие за главным члены в разложении на световом конусе. Явные формулы для них будут приведены позже.

Чтобы яснее представить себе "динамические" сингулярности функционала $G(\xi)$ на световом конусе, вспомним о так называемой “барицентрической” замене $\alpha$-параметров (такие же результаты, конечно, можно получить и при переходе к секторам Хеппа).

Введем вместо переменных $\alpha$ новые переменные

$$
\begin{aligned}
\lambda & =\alpha_{0}+\alpha_{1}+\alpha_{2}+\alpha_{3}, \\
\beta_{0} & =\alpha_{0}\left(\alpha_{0}+\alpha_{1}+\alpha_{2}+\alpha_{3}\right), \\
\beta_{1} & =\alpha_{1}\left(\alpha_{0}+\alpha_{1}+\alpha_{2}+\alpha_{3}\right), \\
\beta_{2} & =\alpha_{2}\left(\alpha_{0}+\alpha_{1}+\alpha_{2}+\alpha_{3}\right) .
\end{aligned}
$$

Обратная замена дается формулами

$$
\alpha_{0}=\lambda \beta_{0}, \quad \alpha_{1}=\lambda \beta_{1}, \quad \alpha_{2}=\lambda \beta_{2}, \quad \alpha_{3}=\lambda\left(1-\beta_{0}-\beta_{1}-\beta_{2}\right) .
$$

Якобиан этой замены есть

$$
\frac{d\left(\alpha_{0}, \alpha_{1}, \alpha_{2}, \alpha_{3}\right)}{d\left(\lambda, \beta_{0}, \beta_{1}, \beta_{2}\right)}=\lambda^{3}
$$

Чтобы не нарушалась симметрия по отношению к нумерации строк, запишем для каждой функции $\Phi\left(\alpha_{0}, \alpha_{1}, \alpha_{2}, \alpha_{3}\right)$ соотношение

$$
\int_{0}^{\infty} d \underline{\alpha} \Phi(\underline{\alpha}) \rightarrow \int_{0}^{\infty} d \lambda \lambda^{3} \int_{0}^{\underline{1}} d \underline{\beta} \delta\left(1-\left[\beta_{0}+\beta_{1}+\beta_{2}+\beta_{3}\right]\right) \Phi\left(\lambda \beta_{0}, \lambda \beta_{1}, \lambda \beta_{2}, \lambda \beta_{3}\right) .
$$

После “барицентрической” замены переменных получим из (32) неравенство

$$
|G(\xi)|<\mathrm{Const} \int_{0}^{\infty} \frac{d \lambda}{\lambda} \int_{0}^{\underline{1}} d \underline{\beta} \frac{\delta\left(1-\sum_{i=0}^{3} \beta_{i}\right)}{\beta_{0}^{2}\left(1-\beta_{0}\right)^{2}} \exp \left\{i \xi^{2} \frac{1}{4 \lambda \beta_{0}\left(1-\beta_{0}\right)}\right\} \exp \{i \lambda E(\underline{\beta})\}
$$

Вводя еще одну новую переменную $\tau=\lambda / \xi^{2}$, получим

$$
\begin{aligned}
|G(\xi)|< & \text { Const } \int_{0}^{\infty} \frac{d \tau}{\tau} \int_{0}^{\underline{1}} \frac{d \underline{\beta}}{\beta_{0}^{2}\left(1-\beta_{0}\right)^{2}} \delta\left(1-\beta_{0}-\beta_{1}-\beta_{2}-\beta_{3}\right) \times \\
& \times \exp \left\{i \tau \xi^{2} E(\underline{\tau})\right\} \exp \left\{\frac{i}{4 \tau \beta_{0}\left(1-\beta_{0}\right)}\right\} .
\end{aligned}
$$

Таким образом, функционал $G(\xi)$ остается ограниченным при $\xi^{2} \rightarrow 0$. Более того,

$$
\begin{aligned}
G(0)= & \text { Const } \int_{0}^{\infty} \frac{d \tau}{\tau} \int_{0}^{\underline{1}} \frac{d \underline{\beta}}{\beta_{0}^{2}\left(1-\beta_{0}\right)^{2}} \times \\
& \times \delta\left(1-\beta_{0}-\beta_{1}-\beta_{2}-\beta_{3}\right) \exp \left\{\frac{i}{4 \tau \beta_{0}\left(1-\beta_{0}\right)}\right\}, \\
\frac{d G}{d\left(\xi^{2}\right)}(0)= & \operatorname{Const} \int_{0}^{\infty} d \tau \int_{0}^{\underline{1}} \frac{d \underline{\beta}}{\beta_{0}^{2}\left(1-\beta_{0}\right)^{2}} \times \\
& \times \delta\left(1-\beta_{0}-\beta_{1}-\beta_{2}-\beta_{3}\right) i E(\underline{\beta}) \exp \left\{\frac{i}{4 \tau \beta_{0}\left(1-\beta_{0}\right)}\right\} .
\end{aligned}
$$


Можно видеть, что

$$
G(0)=G^{\lim }(0), \quad \frac{d G}{d\left(\xi^{2}\right)}(0)=\frac{d G^{\lim }}{d\left(\xi^{2}\right)}(0)
$$

Возврашаясь к остаточному члену $Q(\xi)$, заметим, что он имеет нуль порядка $\left(\xi^{2}\right)^{2}$ при $\xi^{2} \rightarrow 0$. Это дает необходимое обоснование всем нашим построениям.

Наконец, вернемся к разложению на световом конусе. Остается только определить следующую за главной часть разложения (43).

Чтобы упростить громоздкие формулы, введем последнее символическое обозначение для меры интегрирования по $d \tau$ и $d \underline{\beta}$. Обозначим

$$
\begin{aligned}
\int d \mu(\tau, \underline{\beta}) \ldots= & \int_{0}^{\infty} \frac{d \tau}{\tau} \int_{0}^{\underline{1}} \frac{d \underline{\beta}}{\left(\beta_{0}\right)^{2}\left(1-\beta_{0}\right)^{2}} \times \\
& \times \delta\left(1-\beta_{0}-\beta_{1}-\beta_{2}-\beta_{3}\right) \exp \left\{\frac{i}{4 \tau \beta_{0}\left(1-\beta_{0}\right)}\right\} \ldots
\end{aligned}
$$

В этих обозначениях получим

$$
\begin{aligned}
\Psi^{\prime}\left(\xi^{2} \mid \kappa_{1}, \kappa_{2}\right) & =\int d \mu(\tau, \underline{\beta}) \kappa_{1} \xi^{2} \delta\left(\kappa_{1}-\frac{\beta_{2}+\beta_{3}}{1-\beta_{0}}\right) \delta\left(\kappa_{2}-\frac{\beta_{2}}{1-\beta_{0}}\right), \\
\Psi^{\prime \prime}\left(\xi^{2} \mid \kappa_{1}, \kappa_{2}\right) & =\int d \mu(\tau, \underline{\beta}) \kappa_{2} \xi^{2} \delta\left(\kappa_{1}-\frac{\beta_{2}+\beta_{3}}{1-\beta_{0}}\right) \delta\left(\kappa_{2}-\frac{\beta_{2}}{1-\beta_{0}}\right), \\
\Psi_{11}\left(\xi^{2} \mid \kappa_{1}, \kappa_{2}\right) & =\int d \mu(\tau, \underline{\beta}) E_{11} \xi^{2} \delta\left(\kappa_{1}-\frac{\beta_{2}+\beta_{3}}{1-\beta_{0}}\right) \delta\left(\kappa_{2}-\frac{\beta_{2}}{1-\beta_{0}}\right), \\
\Psi_{22}\left(\xi^{2} \mid \kappa_{1}, \kappa_{2}\right) & =\int d \mu(\tau, \underline{\beta}) E_{22} \xi^{2} \delta\left(\kappa_{1}-\frac{\beta_{2}+\beta_{3}}{1-\beta_{0}}\right) \delta\left(\kappa_{2}-\frac{\beta_{2}}{1-\beta_{0}}\right), \\
\Psi_{12}\left(\xi^{2} \mid \kappa_{1}, \kappa_{2}\right) & =\int d \mu(\tau, \underline{\beta}) E_{12} \xi^{2} \delta\left(\kappa_{1}-\frac{\beta_{2}+\beta_{3}}{1-\beta_{0}}\right) \delta\left(\kappa_{2}-\frac{\beta_{2}}{1-\beta_{0}}\right)
\end{aligned}
$$

и

$$
\begin{aligned}
J^{\prime}\left(\kappa_{1}, \kappa_{2} \mid \boldsymbol{\xi}\right) & =:\left.\frac{d \varphi(l)}{d l^{0}}\right|_{l=\kappa_{1} \tilde{\xi}} \varphi\left(\kappa_{2} \tilde{\xi}\right): \frac{1}{2|\boldsymbol{\xi}|}, \\
J^{\prime \prime}\left(\kappa_{1}, \kappa_{2} \mid \boldsymbol{\xi}\right) & =:\left.\varphi\left(\kappa_{1} \tilde{\xi}\right) \frac{d \varphi(n)}{d n^{0}}\right|_{n=\kappa_{2} \tilde{\xi}}: \frac{1}{2|\boldsymbol{\xi}|}, \\
J_{11}\left(\kappa_{1}, \kappa_{2} \mid \boldsymbol{\xi}\right) & =:\left.\frac{d^{2} \varphi(l)}{(d l)^{2}}\right|_{l=\kappa_{1} \tilde{\xi}} \varphi\left(\kappa_{2} \tilde{\xi}\right):, \\
J_{22}\left(\kappa_{1}, \kappa_{2} \mid \boldsymbol{\xi}\right) & =:\left.\varphi\left(\kappa_{1} \tilde{\xi}\right) \frac{d^{2} \varphi(n)}{(d n)^{2}}\right|_{n=\kappa_{2} \tilde{\xi}}: \\
J_{12}\left(\kappa_{1}, \kappa_{2} \mid \boldsymbol{\xi}\right) & =: \frac{d \varphi(l)}{d l} \frac{d \varphi(n)}{d n}:\left.\right|_{l=\kappa_{1} \tilde{\xi} ; n=\kappa_{2} \tilde{\xi}} .
\end{aligned}
$$

Благодарности. Работа была поддержана РФФИ, грант № 01-01-01043. 


\section{Список литературы}

[1] S. A. Anikin, O. I. Zavialov. Ann. Phys. 1978. V. 116. P. 135.

[2] О. И. Завьялов. Перенормированные диаграммы Фейнмана. М.: Наука, 1979.

[3] O. I. Zavialov. Renormalized Quantum Field Theory. Dordrecht: Kluwer Academic Publishers, 1989.

[4] G. G. Callan, D. Gross. Phys. Rev. Lett. 1969. V. 22. P. 156; D. Gross, R. Jackiv. Phys. Rev. D. 1972. V. 6. P. 477; M. E. Peskin, D. V. Schroeder. An Introduction to Quantum Field Theory. Reading, MA: Addison-Wesley, 1995.

[5] Ji Xiangdong. Off-forward parton distribution. hep-ph/9807358; G. R. Katz. Phys. Rev. D. 1985. V. 31. P. 652.

[6] B. Geyer, L. Marcus, D. Robaschik. Nucl. Phys. B. 2001. V. 618. P. 99; J. Blumein. On the structure of the virtual Compton amplitude in the generalized Bjorken region: integral relations. hep-ph/0002071; J. Blumlein, B. Geyer, D. Robaschik. Nucl. Phys. B. 1999. V. 560. P. 283; hep-ph/9903520. 